\title{
Análise da Variabilidade da Temperatura e Precipitação e sua Correlação na Ocorrência da Malária em Quelimane, Moçambique
}

\author{
Analysis of Temperature and Precipitation Variability and its Correlation in the \\ Occurrence of Malaria in Quelimane, Mozambique
}

\section{Análisis de la Variabilidad de la Temperatura y Precipitación y su Correlación en la Ocurrencia de la Malaria en Quelimane, Mozambique}

\author{
Cardenito Mário Colher ${ }^{1}$
}

\begin{abstract}
RESUMO: A malária é uma doença infecciosa causada pelo parasita protozoário unicelular do gênero Plasmodium. É uma patologia que continua a causar significante morbimortalidade nas áreas onde é prevalente, sobretudo em Moçambique. Por se considerar que a malária faz parte de um grupo de doenças tropicais negligenciadas, este artigo desenvolve uma perspectiva voltada para a análise dos determinantes naturais da ocorrência da doença, particularmente no que se refere ao clima (variabilidade da temperatura e da precipitação) na cidade de Quelimane. O período de análise dos dados climáticos corresponde a 37 anos, de 1980 a 2017; e a correlação estabelecida com a malária abrange o período de 17 anos, de 2000 a 2017. Partindo do pressuposto teórico de que os eventos associados à ocorrência da doença estão fortemente relacionados à variação da temperatura e precipitação, por meio do método quantitativo, utilizou-se a técnica estatística dos quantis para identificação dos anos-padrão para analisar as tendências da variabilidade da temperatura e da precipitação. Assim sendo, estabeleceram-se as correlações por intermédio do Coeficiente de Pearson para identificar a correlação entre a temperatura, a precipitação e a malária, sendo que a ocorrência da malária foi consistente com a sazonalidade. Os resultados demostraram que a precipitação tem uma influência imediata - sazonal e anual, ao passo que a temperatura é favorável ao longo de todo o ano na ocorrência da malária em Quelimane.
\end{abstract}

PALAVRAS-CHAVE: Malária. Temperatura. Precipitação. Variabilidade climática. Correlação.

\begin{abstract}
Malaria is an infectious disease caused by unicellular protozoan parasite of Plasmodium genus. It is a condition that continues to cause significant morbidity and mortality in areas where it is prevalent, especially in Mozambique. Because malaria is considered to be part of a neglected tropical diseases group, this article develops a perspective on the analysis of natural determinants of malaria occurrence, particularly with regard to climate (temperature variability and precipitation) in Quelimane city. The analysis of climate data corresponds to a period of 37 years, from 1980 to 2017; and the correlation established with malaria covers the period of 17 years, from 2000 to 2017. Based on the theoretical assumption that the events associated with occurrence of this disease are strongly related to temperature and precipitation variation, using the quantitative method, the statistical technique of quantiles was used to identify standard years in order to analyze the trends of temperature variability and precipitation. Therefore, correlations were established using Pearson's Coefficient to identify the
\end{abstract}

\footnotetext{
${ }^{1}$ Universidade Federal do Paraná. Av. Cel. Francisco H dos Santos, 100 - Ed. João José Bigarella - 1ํandar sala 108, Jardim das Américas, Curitiba, CEP: 81531-980. cardenitocolher@yahoo.com.br.
} 
correlation between temperature, precipitation and malaria, and the occurrence of malaria was consistent with seasonality. The results showed that precipitation has an immediate influence seasonal and annual, while the temperature is favorable throughout the year in the occurrence of malaria in Quelimane.

KEYWORDS: Malaria. Temperature. Precipitation. Climate variability. Correlation.

RESUMEN: La malaria es una enfermedad infecciosa causada por el parásito protozoario unicelular del género Plasmodium. Es una condición que continúa causando morbilidad y mortalidad significativas en áreas donde prevalece, especialmente en Mozambique. Debido a que la malaria se considera parte de un grupo de enfermedades tropicales desatendidas, este artículo desarrolla una perspectiva sobre el análisis de los determinantes naturales de la aparición de la malaria, particularmente con respecto al clima (variabilidad de temperatura y precipitación) en la ciudad de Quelimane. El período de análisis de datos climáticos corresponde a 37 años, de 1980 a 2017; y la correlación establecida con la malaria cubre el período de 17 años, desde 2000 hasta 2017. Basado en el supuesto teórico de que los eventos asociados con la aparición de la enfermedad están fuertemente relacionados con la variación de temperatura y precipitación, por medio del método cuantitativo, se utilizó la técnica estadística de los cuantiles para la identificación de años estándar para analizar las tendencias de la variabilidad de la temperatura y la precipitación. Por lo tanto, se establecieron correlaciones usando el Coeficiente de Pearson para identificar la correlación entre temperatura, precipitación y malaria, y la aparición de malaria fue consistente con la estacionalidad. Los resultados mostraron que la precipitación tiene una influencia inmediata: estacional y anual, mientras que la temperatura es favorable durante todo el año en la aparición de malaria en Quelimane.

PALABRAS-CLAVES: Malaria. Temperatura. Precipitación. Variabilidad climática. Correlación.

\section{INTRODUÇÃO}

A malária é uma doença infeto-parasitária que abrange um quarto da população mundial e por norma é responsável pela morte de cerca de um milhão e meio a três milhões de pessoas a cada ano. Por esta razão, a (OMS) Organização Mundial da Saúde considera esta doença como sendo um dos maiores problemas de saúde pública na atualidade, particularmente nos "países terceiro mundistas" ou em vias de desenvolvimento com ênfase para os da África Subsaariana, como é o caso de Moçambique (CORDEIRO et al., 2002).

Dados apresentados pela Organização Mundial da Saúde dão conta de que foram registados duzentos e sete milhões de casos de malária e que $90 \%$ das mortes por malária ocorreram na África Subsaariana, onde 77\% destas mortes ocorrem em crianças menores de cinco anos de idade (WORLD HEALTH ORGANIZATION, 2013).

Segundo França, Santos e Figueroa-Villar (2008), esta doença é considerada uma das mais antigas e mortais transmitidas para os humanos através de vetores do género Anopheles ( $A n)$ e o ciclo de transmissão é essencialmente determinada pela "picada" do mosquito fêmea em seres humanos. Os eventos associados à ocorrência da malária estão fortemente relacionados a fatores climáticos (variação da temperatura e precipitação, particularmente), por serem considerados essenciais para a ocorrência e para o 
desenvolvimento do vetor e do parasita, bem como relacionados aos fatores socioeconômicos.

Para o caso moçambicano, pela sua localização geográfica o país tem sofrido sazonalmente em intervalos anuais a ação dos eventos extremos. Vale ressaltar que, através de resultados de estudos feitos por Epstein (2009), Mabunda (2006), Queface e Tadross (2009) e pelo (MICOA) Ministério para Coordenação da Ação Ambiental, a ocorrência dos referidos eventos extremos pode estar associada com as mudanças climáticas que ocorrem no país. (MOÇAMBIQUE, 2004)

Estes eventos têm se manifestado através de chuvas intensas, criado inundações nos rios e lagos e também criando corpos de água em unidades residenciais e destruido e inundado habitações, fazendo com que a população passe a viver temporariamente em "centros de acolhimento", aumentando cada vez mais a sua vulnerabilidade e o risco de contrair a malária.

Em Moçambique, com destaque para a cidade de Quelimane, o quadro epidemiológico é largamente dominado por doenças transmissíveis como a malária - principal causa de morte no país - e por doenças infecciosas causadas pela proliferação do agente etiológico tanto da malária quanto da cólera e de outras doenças de origem hídrica. Por norma, autores como Mabunda (2006) consideram que a malária constitui um dos principais problemas de saúde pública em Moçambique devido à existência de condições favoráveis para criadouros do vetor responsável para a transmissão da doença.

Os dados apresentados pelo (MISAU) Ministério da Saúde em Moçambique, do nível central ao local e de Organizações Não Governamentais (ONG's) que trabalham com esta questão considerarem esta doença como a principal causa dos problemas de saúde, sendo responsável por $40 \%$ de todas as consultas externas. Ainda, até $60 \%$ de doentes internados nas enfermarias de pediatria são admitidos como resultado da malária severa. A malária é também a principal causa de mortalidade nos hospitais em Moçambique, representando $30 \%$ de todos os óbitos registados. A estimativa de prevalência no grupo etário de 2 a 9 anos de idade varia de 40 a $80 \%$, com $90 \%$ de crianças menores de cinco anos de idade infectadas por parasitas da malária em algumas áreas (MABUNDA, 2006; MENENDEZ, 1995; MOÇAMBIQUE, 2006; SAÚTE et al., 2003).

Por isso, considerando que de acordo com a World Health Organization (2014) a malária faz parte de um grupo de doenças tropicais negligenciadas, o artigo pretende trazer contribuições no campo de estudos relacionados com a geografia da saúde, sobretudo com os fatores climáticos e sua influência na ocorrência da doença, visto que este campo tem chamado atenção dos acadêmicos e também de entidades governamentais da sociedade. Pretende de igual forma analisar os aspetos socioambientais associados à ocorrência de doenças, procurando trazer contributos teóricos atinentes aos fatores geofisicos que se 
relcionam aos eventos da malária. As análises neste artigo estão voltadas para a variabilidade da temperatura e precipitação e sua correlação na ocorrência da malária em Quelimane/Moçambique, por considerar que as pesquisas desenvolvidas em Moçambique não têm explorado a influência dos fatores ambientais ou climáticos na ocorrência da doença.

\section{MÉTODO - TÉCNICAS DE PESQUISA}

O estudo foi desenvolvido em Moçambique, na cidade Quelimane, que é a capital da província da Zambézia (Figura 1). Localiza-se a cerca de 20 quilômetros do Oceano Índico, entre as coordenadas $16^{\circ} 51^{\prime}$ '00"S e 3659' 00"E e tem um clima tropical úmido. Os dados climáticos deste artigo foram obtidos no (INAM) Instituto Nacional de Meteorologia de Moçambique (2018) - Delegação Provincial da Zambézia em Quelimane e os dados da malária foram obtidos através da Direção Provincial de Saúde da Zambézia - Direção Distrital de Saúde de Quelimane. (MOÇAMBIQUE, 2018).

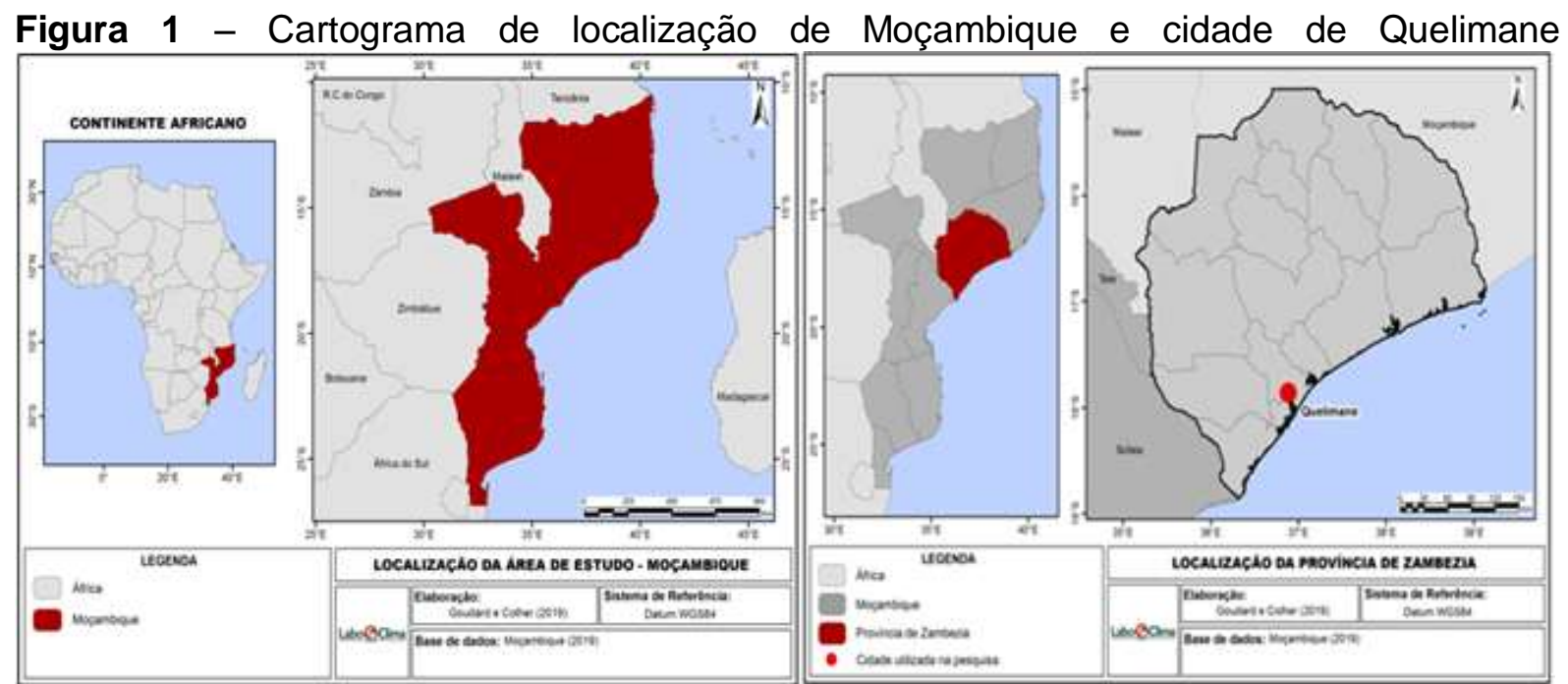

Fonte: organizado pelo autor.

Para a análise da variabilidade e identificação das tendências na cidade em estudo, utilizou-se o método dos anos-padrão por intermédio da técnica estatística dos quantis, procedimento utilizado por Xavier e Xavier (1999), Xavier, Silva e Rebelo (2002) para definir classes de precipitação. Neste artigo este processo consistiu em empregar os percentis dos totais anuais da precipitação e da temperatura média anual, e os mesmos foram ordenados de maneira crescente, definidos de acordo com as seguintes classes: muito seco para os $10 \%$ menores valores, seco entre os $10 \%$ e $35 \%$ das observações, normal entre os $35 \%$ e $65 \%$, chuvoso entre os $65 \%$ e $90 \%$ e muito chuvoso para os valores acima de $90 \%$ (SILVESTRE; LIMA NETO; FLORES, 2013). 
É importante salientar que o critério para a definição das classes acima apresentadas seguiu o mesmo preceito que para os valores da temperatura média anual, destancando-se para o primeiro grupo de anos-padrão as temperaturas frias ou baixas, seguidas das tendentes a frias ou baixas, em terceiro lugar encontram-se as normais, e a seguir as tendentes a quentes ou altas e, finalmente, as temperaturas quentes.

Ainda utilizou-se o programa Excel para a produção de planilhas, o que consequentemente possibilitou a geração de tabelas e gráficos representativos das condições anuais da precipitação e da temperatura.

Para a avaliação da precipitação, neste artigo, foram considerados os totais anuais e mensais ao longo da série histórica, por um lado e, por outro, com relação à temperatura, consideraram-se as médias das máximas e mínimas na escala anual e mensal. Todavia, para o caso da temperatura, na análise das tendências fundamentada no método dos anospadrão levaram-se em conta os valores da temperatura média anual.

Efetuaram-se de igual forma cálculos para se estabelecer a análise da correlação entre os casos de malária e a variabilidade da temperatura e precipitação. Os dados de malária foram obtidos através de uma escala mensal numa série de 17 anos, ou seja, de 2000 a 2017. A correlação foi analisada por meio do Coeficiente de Correlação de Pearson, de forma a verificar se uma modificação na variável precipitação e temperatura terá um impacto equivalente na variável na malária, medindo a associação linear entre ambas, que é uma medida da variância comum entre duas variáveis (FIGUEIREDO FILHO; SILVA JÚNIOR, 2009).

A correlação foi determinada através da estimativa do Coeficiente de Pearson que é tido como uma medida do grau de relação linear entre duas variáveis quantitativas, descrita através da seguinte formula:

$$
y_{i}=\beta_{0}+\beta_{1} x_{i}+\epsilon_{i}, \quad i=1, \ldots, n,
$$

Onde: yi é o valor da variável dependente (resposta) para o i-ésimo elemento da amostra;

xi: valor (conhecido) da variável independente ou preditora para o i-ésimo elemento da amostra;

$\beta 0$ e $\beta 1$ : são parâmetros desconhecidos;

Ei: erro amostral (PETERNELLI, 2015).

A significância estatística da análise das correlações acima descritas foi calculada através de um teste baseado na distribuição teste $t$ de Student de acordo com a seguinte equação: 


$$
t=\frac{\bar{x}-\mu}{\frac{s}{\sqrt{72}}}
$$

Onde: $\overline{\mathcal{X}}$ é a média da amostra;

$\mu$ é a média da população (ou referência);

S é o desvio padrão;

n é o número de sujeitos (VILELA JUNIOR, 2019).

\section{ASPECTOS SOBRE A VARIABILIDADE CLIMÁTICA E A MALÁRIA}

Os estudos sobre a variabilidade climática e a malária se enquadram na ciência geográfica num contexto em que há reestruturação das suas áreas de atuação, tendo as relações espaciais como uma categoria de análise. Foi surgindo um pensamento dentro da geografia humana, uma ramificação que olhava para os processos de saúde e de doença - a geografia da saúde, e que se caraterizava pela particularidade de observar a distribuição espacial das enfermidades podendo ter ou não relação com os aspetos geofisiconaturais e geosociais (COSTA; TEIXEIRA, 1999; LIMA NETO, 2000).

Segundo Peiter (2007), entre os principais objetivos deste campo do conhecimento destaca-se aquele que procura proporcionar novos conhecimentos e desenvolver uma proposta teórico-metodológica para o estudo das relações geoespaciais dos processos de saúde e de doença da população e o aprimoramento de pesquisas tendentes à busca de soluções para as doenças consideradas negligenciadas, como a malária, através de explicações e abordagens locais, para melhorar o bem-estar da população.

Este bem-estar resulta da interação entre os fatores sóciopoliticos, socioeconômicos, socioculturais e sócioambientais no qual o clima, por intermédio da sua variabilidade, tem exercido uma influência como fator determinante e limitante para a saúde e para a ocorrência da malária. Por isso, esta persperctiva dentro da geografia da saúde é desenvolvida através da abordagem associada à relação entre o clima e a saúde e, no caso deste artigo, entre a variabilidade da temperatura e da precipitação e a malária.

Vários estudos a respeito do clima ressaltam e dão ênfase ao fenômeno das mudanças climáticas, porém, considerando que se trata de um evento complexo, estudar a sua variação torna-se fundamental para a sua compreensão, ainda que a variabilidade seja uma componente conhecida da dinâmica climática, o seu impacto mesmo "[...] dentro dos limites esperados [...]" pode ter reflexos significativos nas mais diversas atividades humanas e nos processos de saúde e de doença (NUNES; LOMBARDO, 1995, p. 21). 
Buscando as palavras de Christofoletti (1992), a variabilidade climática pode ser entendida como a forma por qual os valores de um elemento climático variam no interior de um determinado período, ou seja, numa série temporal.

Já Confalonieri (2003) considera a variabilidade climática como a propriedade intrínseca do sistema climático terrestre, responsável por oscilações naturais nos padrões climáticos, observados em todos os níves da escala espacial. Inclui as variações sazonais, bem como variações em grande escala na circulação atmosférica e oceânica, como o El Niño/Oscilação Sul - ENOS. Em geral, as mudanças no clima ocorrem como resultado da variabilidade interna do sistema climático e de fatores externos (naturais e antropogênicos).

Segundo Uele (2013), para o caso da África Austral, na qual Moçambique se localiza, de forma geral nesta região destacam-se a influência dos modos de variabilidade climática como o El Niño - Oscilação Sul, Oscilação Decadal do Pacífico, o Dipolo do Oceano Índico, a Zona de Convergência Intertropical, a Zona de Convergência do Oceano Índico e os sistemas de baixas pressões associadas ao cavado equatorial. Os efeitos no clima mais conhecidos e de maiores impactos provocados pelo ENOS estão relacionados à variabilidade no regime térmico e, principalmente, no padrão de distribuição da chuva em Moçambique.

Vários estudos têm abordando a temática da variabilidade climática (BRAGANZA et al., 2003; CHRISTOFOLETTI, 1992; CONFALONIERI, 2003; NUNES; LOMBARDO, 1995; QUEIROZ; COSTA, 2012) porém ainda se está longe de chegar a um consenso, principalmente no que tange à influência local nas tendências globais. Todavia, os estudos de variação do clima local, principalmente sobre a temperatura, a precipitação e a umidade relativa, são essenciais para se compreender os impactos que estas variações podem trazer para a população como um todo. Por isso, tem se considerado que variações significativas em escala ou no âmbito local poderão causar impactos socioeconômicos e na proliferação de vetores de doenças.

A respeito da proliferação de doenças infecciosas e parasitárias, por meio de estudos e conhecimento sobre a variabilidade climática é possível a compreensão da distribuição espacial e temporal da prevalência de doenças como a malária, por exemplo, e das variáveis associadas ao clima nas áreas endêmicas (BAUTISTA et al., 2006; GERMPERLI et al., 2006).

Apesar de alguns estudos sustentarem que o conjunto de dados climáticos geralmente é utilizado para explicar a variação espacial, sazonal e interanual da prevalência de doenças como a malária na África, por exemplo, muitas vezes não há consenso sobre a importância relativa dos diferentes fatores envolvidos (MABASO et al., 2006; THOMSON et al., 2005). Todavia, é de notar que estas variações climáticas exercem fortes influências nas distribuições geográficas da prevalência de doenças parasitárias. 
Um estudo realizado por Adu-Prah e Koffi (2015) em Gana, demonstrou que para a compreensão da prevalência da malária ao longo do tempo foi necessário estudar a variabilidade climática e os seus impactos na prevalência da doença. Já em Bangladesh, a pesquisa desenvolvida por Sutherst (2004) concluiu que as tendências nos fatores climáticos constituem uma das forças motrizes que afetam de forma significativa a transmissão da malária.

Ainda na mesma temática, pesquisas realizadas em várias partes do mundo (Sri Lanka, Quênia, Índia, Ruanda e Malaui), correlacionaram a variabilidade climática com a incidência de malária através da influência que os padrões de chuva, temperatura e umidade exerciam nos níveis de propagação da doença (BHATTACHARYA et al., 2006; BRIËT et al., 2008; HASHIZUME; TERAO; MINAKAWA, 2009). Por isso, para o caso de estudo de surtos de doenças quer sejam em escalas sazonais ou interanuais, a análise da variação do clima torna-se fundamental.

A respeito da malária, segundo Webber (2004), considera-se que é uma doença transmitida pela "picada" de mosquito fêmea apesar de existirem espécies mais eficazes que outras na transmissão de doença. Por norma, esta eficácia depende de fatores como: a fonte de alimento que pode ser humana ou outra, o local em que vive (dentro ou fora das residências), o momento em que se alimenta e o tempo de vida do mosquito.

Os fatores que devem ser considerados na ocorrência desta doença são as condições ambientais, sobretudo a variação da temperatura e precipitação. Em relação à temperatura, apesar da elevada resistência a temperaturas elevadas, o comportamento do mosquito é muito sensível a este fator, podendo afetar aspectos como a duração do período de hibernação e o estímulo à alimentação. Para além de afetar o comportamento do mosquito, condiciona o desenvolvimento do parasita no interior do mosquito (CAPINHA, 2009; IMPOINVIL et al., 2007; WEBBER, 2004).

O outro aspecto ou fator ambiental associado à malária que deve ser considerado é a precipitação pluvial ou a presença de corpos de água em locais de reprodução. Geralmente, a água fornece um habitat para os mosquitos depositarem seus ovos e para 0 desenvolvimento do seu ciclo de vida. A presença de corpos de água permanentes condiciona a existência de criadouros de mosquitos e a possibilidade de transmissão da malária ao longo do ano (ERNST et al., 2006; OESTERHOLT et al., 2006; SNOW; GILLES, 2002). 


\section{RESULTADOS E DISCUSSÃO}

\section{Variabilidades Inter-anual da Temperatura}

Analisando os dados da variabilidade inter-anual da temperatura na cidade de Quelimane, por intermédio de procedimentos e técnicas estatísticas previamente apresentadas, com fundamentos nos anos-padrão, nota-se que a maior parte dos anos da série em análise foi normal: 1983, 1987, 1989, 1992, 1996, 1997, 1998, 2002, 2003, 2007 e 2012.

De forma geral, os dados da Tabela 1 demostram que os anos frios ou com temperaturas baixas foram os de 1981, 1985, 1991 e 1995. Os tendentes a frio: 1980, 1982, 1984, 1986, 1988, 1990, 1994, 1999 e 2000. Já os tendentes a quente pertencem aos anos: 1993, 2001, 2004, 2006, 2008, 2009, 2013, 2014, 2015 e 2017. Finalmente, os anos quentes foram os de 2005, 2010, 2011 e 2016.

Tabela 1 - Variabilidade Inter-anual da Temperatura Média em Quelimane - 1980 a 2017

\begin{tabular}{|c|c|c|c|c|c|c|}
\hline Classes & & Frio/ baixa & $\begin{array}{l}\text { Tendente a } \\
\text { Frio/Baixa }\end{array}$ & Normal & $\begin{array}{l}\text { Tendente a } \\
\text { Quente/Alta }\end{array}$ & Quente/Alta \\
\hline \multirow{10}{*}{$\begin{array}{l}\text { Anos- } \\
\text { Padrão }\end{array}$} & & 1995 & 1982 & 1989 & 2001 & 2016 \\
\hline & & 1985 & 1986 & 1992 & 2004 & 2005 \\
\hline & & 1991 & 1988 & 1997 & 2013 & 2010 \\
\hline & & 1981 & 1980 & 1998 & 2009 & 2011 \\
\hline & & & 1994 & 1996 & 2014 & \\
\hline & & & 2000 & 1983 & 2008 & \\
\hline & & & 1984 & 2002 & 1993 & \\
\hline & & & 1999 & 2012 & 2017 & \\
\hline & & & 1990 & 1987 & 2015 & \\
\hline & & & & 2007 & 2006 & \\
\hline \multirow{5}{*}{$\begin{array}{l}\text { Análise } \\
\text { Estatística }\end{array}$} & & & & 2003 & & \\
\hline & Média & 23,92840909 & 24,67651515 & 25,3147 & 25,7955 & 26,36145833 \\
\hline & Variância & 0,13171105 & 0,065663417 & 0,02932 & 0,015710409 & 0,021532118 \\
\hline & $\begin{array}{l}\text { Desvio } \\
\text { Padrão }\end{array}$ & 0,36292017 & 0,25624874 & 0,17122 & 0,12534117 & 0,146738264 \\
\hline & $\begin{array}{l}\text { Coeficiente } \\
\text { de variação }\end{array}$ & 1,516691598 & 1,038431637 & 0,67635 & 0,48590324 & 0,556639401 \\
\hline
\end{tabular}

Fonte: organizado pelo autor.

$\mathrm{Na}$ relação destes dados com os surtos, por meio da Figura 2, observa-se que nos anos tendentes a quentes como: 2006, 2009 e 2017, por exemplo, e nos anos quentes, como é o caso de 2005 e 2016, as taxas de inicidência atingiram os valores mais elevados, variando de 20 a $29 \%$ do total da população, demonstrando que o aumento da temperatura influencia os casos da doença. 
Figura 2 - Gráfico de variação da temperatura anual e mensal na cidade de Quelimane - 1980 a 2017

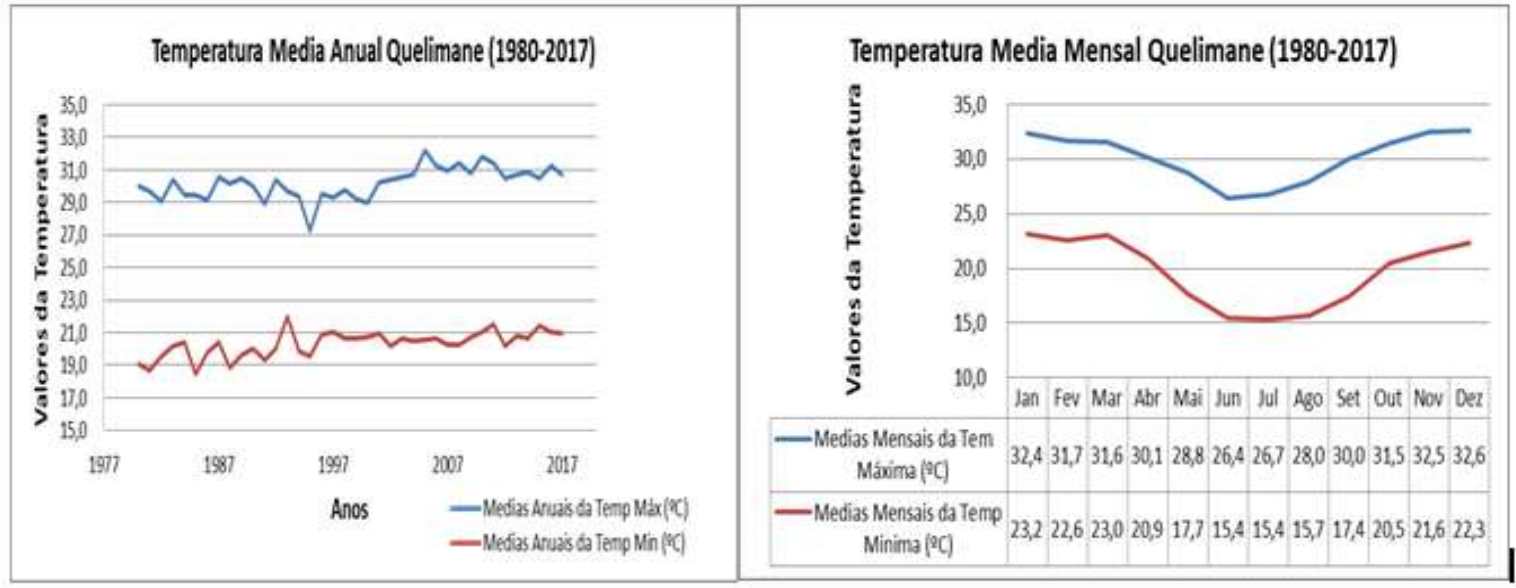

Fonte: organizado pelo autor.

O exame estatístico destes dados demonstrou que, para o caso dos anos frios, a média foi de $23,9^{\circ} \mathrm{C}$, a variância correspondeu a 0,13 e a extensão da variabilidade em relação à média foi de 1,51 . Já para os anos tendentes a frio, a sua média foi de $24,7^{\circ} \mathrm{C}$, com variância mais reduzida em relação aos anos frios, ou seja, 0,06 e coeficiente de variação de 1,03. Para os anos habituais, que fazem parte do maior conjunto, a média foi de $25,3^{\circ} \mathrm{C}$, que coincide com a média climatológica da cidade de Quelimane, com menor resultado de variância em relação aos anteriores conjuntos e o coeficiente de variação correspondente a 0,67.

Todavia, os dois últimos conjuntos, os anos tendentes a quentes, tiveram a média de $25,7^{\circ} \mathrm{C}$, com variância de 0,015 e a extensão da variabilidade equivalente a 0,48 . Por fim, os anos quentes apresentaram a média de $26,3^{\circ} \mathrm{C}$, valor acima da média climatológica, com a variância de 0,02 e o coeficiente de variação proporcional a 0,55.

De forma geral, nota-se que ao longo dos 37 anos em análise os dados demostraram que a temperatura registrou maior variabilidade nos anos frios e tendentes a frios, pouca variabilidade nos anos tendentes a quentes e variabilidade moderada nos anos quentes. Todavia, os anos tiveram orientação de normal para tendentes ao quente.

A análise da variabilidade da temperatura ocorrida de 1980 a 2017, na escala anual, na série apresentada na Figura 2, revela que houve muita variação. A temperatura máxima variou de $27^{\circ} \mathrm{C}$ a aproximadamente $33^{\circ}$ - registrados em 1995 e 2005. De maneira geral, nos primeiros 10 anos da série, a média da temperatura máxima variou de $28^{\circ} \mathrm{C}$ a $30^{\circ} \mathrm{C}$.

Através dos dados da figura em questão, nota-se que, a partir de 1991 até 2001, registrou-se ascensão da temperatura em relação ao período anterior com variação anual significativa. Em relação aos períodos de 2001 a 2017, a subida observada anteriormente fez com que se registrasse a manutenção da temperatura que oscilava dos $30^{\circ} \mathrm{C}$ aos $33^{\circ} \mathrm{C}$ com tendências para aumento. 
Analisando o mesmo gráfico, a temperatura mínima apresentou variação entre $18^{\circ} \mathrm{C}$ e $22^{\circ} \mathrm{C}$ ao longo da série - registrados em 1985 e 1993. Nos primeiros dez anos a temperatura mínima variou bastante voltando a se estabilizar de 1996 a 2008. A partir de 2009, os últimos anos da série analisada, a temperatura começou a apresentar variação considerável, ou seja, verificou-se instabilidade.

Todavia, nota-se por meio da Figura 2 que, no gráfico representado à direita, sobre a variação da temperatura mensal (máxima e mínima), os meses de junho e julho apresentam as temperaturas mais baixas, chegando a atingir os $15^{\circ} \mathrm{C}$ ao longo deste período e os meses de dezembro e janeiro são os mais quentes apresentando valores correspondentes a $33^{\circ} \mathrm{C}$.

\section{Variabilidades Inter-anual da Precipitação}

A respeito da variabilidade inter-anual, a análise da frequência da precipitação durante os 37 anos em estudo demostrou que a terceira classe, correspondente aos valores superiores a $35 \%$ e iguais ou inferiores a $65 \%$, considerados anos normais, apresentam maiores proporções de ocorrência de acordo com a Tabela 2.

Tabela 2 - Variabilidade Inter-Anual da Precipitação em Quelimane - 1980 a 2017

\begin{tabular}{|c|c|c|c|c|c|c|}
\hline Classes & & Muito seco & seco & Normal & Chuvoso & $\begin{array}{l}\text { Muito } \\
\text { chuvoso }\end{array}$ \\
\hline \multirow{11}{*}{ Anos-Padrão } & & 1999 & 2012 & 2005 & 2009 & 2003 \\
\hline & & 1995 & 1994 & 1988 & 1982 & 1981 \\
\hline & & 1993 & 1985 & 1987 & 1997 & 2017 \\
\hline & & 1989 & 1992 & 1998 & 2014 & 2007 \\
\hline & & & 1991 & 1986 & 1980 & \\
\hline & & & 2001 & 2006 & 1996 & \\
\hline & & & 1990 & 2015 & 2013 & \\
\hline & & & 1983 & 2011 & 1984 & \\
\hline & & & 2010 & 2002 & 2004 & \\
\hline & & & & 2016 & & \\
\hline & & & & 2008 & & \\
\hline \multirow{5}{*}{$\begin{array}{l}\text { Análise } \\
\text { Estatística }\end{array}$} & & & & 2000 & & \\
\hline & Média & 310,47 & 506,3511 & 814,687 & 1111,63 & 1555,365 \\
\hline & Variância & 5788,362733 & 13721,73 & 5634,62 & 20587,1 & 40109,92977 \\
\hline & Desvio Padrão & 76,0812903 & 117,1398 & 75,0641 & 143,482 & 200,2746359 \\
\hline & $\begin{array}{l}\text { Coeficiente de } \\
\text { variação }\end{array}$ & 24,50519867 & 23,1341 & 9,21386 & 12,9074 & 12,87637538 \\
\hline
\end{tabular}

Fonte: organizado pelo autor.

De forma geral, os anos de 1989, 1993, 1995 e 1999 representam os anos muito secos; os de 1983, 1985, 1990, 1991, 1992, 1994, 2001, 2010 e 2012 são os anos secos. Por outro lado, os anos chuvosos são: 1980, 1982, 1984, 1996, 1997, 2004, 2009, 2013 e 
2014, sendo os anos muito chuvosos equivalentes a 1981, 2003, 2007 e 2017 . Os restantes dos anos representam os habituais segundo dados da Tabela 2.

A relação dos dados acima apresentados com os surtos constata-se que, no geral, os anos normais, chuvosos e muito chuvosos, sobretudo anos como 2006, 2008, 2009, 2016 e 2017, por exemplo, apresentam altas taxas de incidência da doença, o que demonstra a influência da precipitação na ocorrência da malária.

Com a observação estatística destes dados, constatou-se que os anos muito secos tiveram a média de 310,47 milímetros com a variância de 5788,36 e o coeficiente de variação equivalente a 24,50. Para os anos secos, a média foi de 506,35 milímetros com a extensão da variabilidade em relação à média igual a 23,13 e variância de 13721,73. Já nos anos habituais, que correspondem ao grupo de maior ocorrência, a média foi proporcional a 814,68 milímetros, abaixo do normal climatológica, com a variância de 5634,62 e o coeficiente de variação de 9,21.

Apesar de o quarto conjunto apresentar a extensão da variabilidade maior em relação aos anos normais, a média dos anos chuvosos está abaixo da normal climatológica, e os anos muito chuvosos têm a média equivalente a 1555,36 milímetros, um valor acima da normalidade climatológica, com o coeficiente de variação igual a 12,87.

Analisando a variabilidade da precipitação pluviométrica ocorrida no período em questão, na escala anual, conforme a informação contida na Figura 3, nota-se que se registrou dinâmica considerável. No período de 1980 a 198, verificou-se em linhas gerais a instabilidade no comportamento pluviométrico, variando em valores de 410 a 1450 milímetros.

Figura 3 - Variação da Precipitação Anual e Mensal em Quelimane - 1980 á 2017

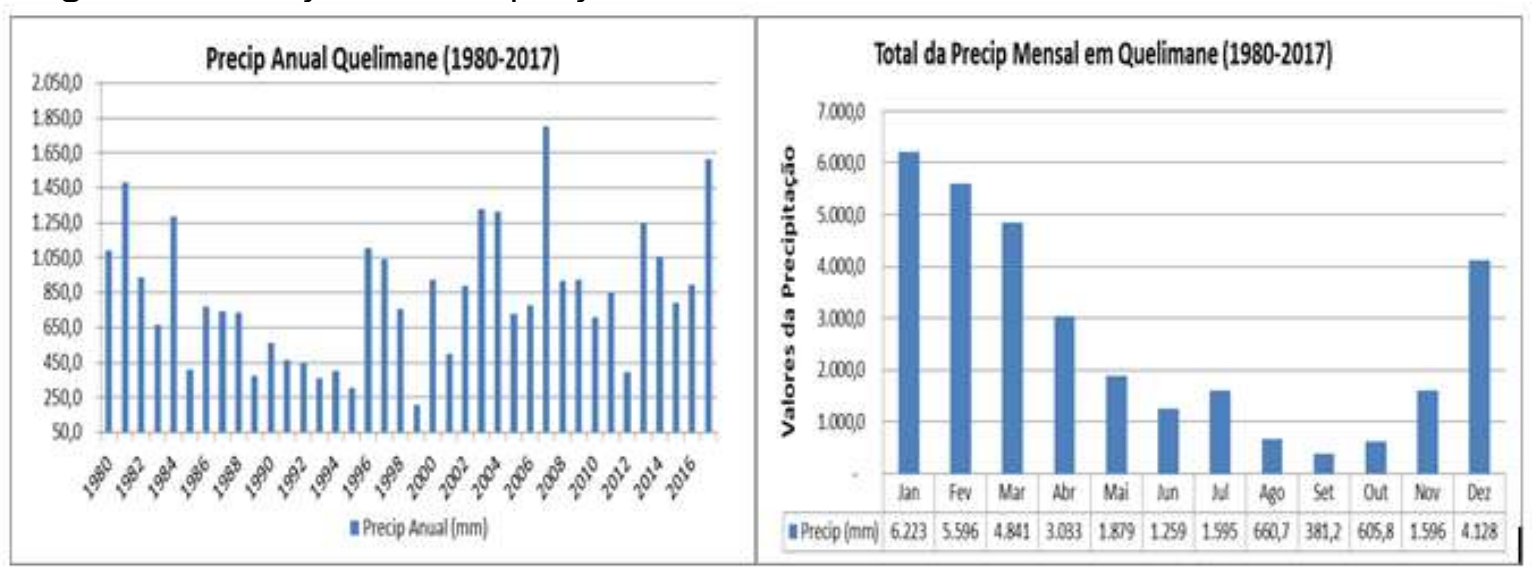

Fonte: organizado pelo autor.

Por outro lado, ainda de acordo com a figura a seguir, observando os últimos 20 anos, período entre 1996 a 2017, a precipitação apresentou variabilidade considerável porque os seus valores oscilavam de 700 (que pode ser considerada a média histórica do período em 
causa) a 1700 milímetros anuais - valor registrado em 2007, apesar de que em 1999 o valor anual da precipitação foi inferior pela tendência de redução da queda pluviométrica.

Com relação ao gráfico à direita, representando o total da precipitação mensal durante os 37 anos analisados, observa-se que a época chuvosa tem o seu início no mês de novembro, tendo se estendido até o mês de abril a maio. O cumulativo da precipitação mostra que os meses de janeiro, fevereiro e março são os mais chuvosos. Por outro lado, a época seca tem o seu início desde os meses de abril a maio sendo que os meses de agosto, setembro e outubro são os que apresentaram os menores valores cumulativos da precipitação, dados que são confirmados no estudo realizado por Sitoe (2017).

\section{Correlações Entre os Dados Climáticos e a Malária}

A respeito deste ponto, calculou-se a correlação entre os valores médios da temperatura e o número de casos de malária, tendo em conta a sazonalidade, ou seja, a correlação da estação/sazonal e a correlação mensal. Observando os dados na escala mensal, no período em estudo, de 2000 a 2017, constatou-se que é positiva, com valores elevados nos meses de dezembro, fevereiro e março, o que coincide com a época chuvosa.

Por outro lado, para os dados sazonais, os valores da Tabela 3 tendem a ser estatisticamente significativos em relação aos dados mensais, o que indica a influência da temperatura no aumento da malária tendo em conta a sazonalidade. Ademais, correlacionando a taxa de incidência da malária com a temperatura, para o período em análise, a correlação tende a ser mais signficativa.

Tabela 3 - Correlação entre Temperatura e Malária - Estação Chuvosa

\begin{tabular}{l|l|l|l|l|l|c|c}
\hline & Nov & Dez & Jan & Fev & Mar & $\begin{array}{l}\text { Correlação da } \\
\text { Estacção }\end{array}$ & $\begin{array}{l}\text { Correlação Taxa de } \\
\text { Inc e Precip }\end{array}$ \\
\hline $\begin{array}{l}\text { Correlação } \\
\text { Mensal }\end{array}$ & 0,03 & 0,21 & $-0,06$ & 0,39 & 0,33 & 0,30 & 0,38 \\
\hline T Student & 0,14 & 0,87 & $-0,23$ & 1,67 & 1,39 & 1,26 & 1,63 \\
\hline
\end{tabular}

Fonte: organizado pelo autor.

Outra vertente de análise a respeito da correlação da temperatura com a malária está relacionada com a estação seca. que é constituída por quatro meses: maio, junho, julho e agosto, meses que, geralmente, na cidade de Quelimane, fazem parte da estação seca e estão relacionados com a época do ano em que os casos de malária têm atingido valores baixos.

Através da Tabela 4 constatou-se que, para a época seca, o mês de agosto apresenta uma correlação negativa, porém os meses de maio, junho e julho demostram que quanto menor for a temperatura, menor são os casos de malária. Por isso, tanto na variação mensal 
como no resultado da correlação sazonal, os valores são pouco expressivos, ou seja, não apresentam uma correlação forte. Este fato se deve não apenas pela escala de dados disponível para realizar o estudo, mas também pelo fato da variação da temperatura exercer influências na ocorrência da malária.

Tabela 4 - Correlação entre Temperatura e Malária - Estação Seca

\begin{tabular}{l|l|l|l|l|l}
\hline & Maio & Jun & Jul & Ago & Correlação da Estacção \\
\hline Correlação Mensal & 0,22 & 0,03 & 0,08 & $-0,01$ & 0,01 \\
\hline T Student & 0,92 & 0,12 & 0,33 & $-0,05$ & 0,02 \\
\hline
\end{tabular}

Fonte: organizado pelo autor.

Ainda no âmbito da correlação a respeito da precipitação, a Tabela 5 apresenta a correlação estatística entre a variação sazonal da precipitação e a malária em Quelimane no período em referência, ou seja, 17 anos. Calculou-se a correlação considerando os mesmos critérios utilizados para o caso da temperatura, considerando apenas os meses da estação chuvosa. De acordo com os dados da tabela, todos os meses da estação apresentam correlação positiva, mas pouco expressivas, devido à escala de dados utilizada, o que significa que, na estação chuvosa, a precipitação exerce influência nos casos de malária.

Tabela 5 - Correlação entre Precipitação e Malária - Estação Chuvosa

\begin{tabular}{l|c|c|c|c|c|c|c}
\hline & Nov & Dez & Jan & Fev & Mar & $\begin{array}{l}\text { Correlação da } \\
\text { Estacção }\end{array}$ & $\begin{array}{l}\text { Correlação Taxa de Inc e } \\
\text { Precip }\end{array}$ \\
\hline $\begin{array}{l}\text { Correlação } \\
\text { Mensal }\end{array}$ & 0,46 & 0,20 & 0,30 & 0,02 & 0,25 & 0,28 & 0,37 \\
\hline T Student & 2,07 & 0,80 & 1,24 & 0,09 & 1,05 & 1,17 & 1,58 \\
\hline
\end{tabular}

Fonte: organizado pelo autor.

Analisando os dados sazonais, os resultados tendem a ser mais significativos, o que explica a influência dos corpos de água como resultado da queda pluviométrica por um período longo. Os dados da correlação entre a incidência da malária com a precipitação tendem de igual forma a apresentar valores cada vez mais significativos estatisticamente, o que explica que a presença da água é um fator de ocorrência e da incidência da malária.

Geralmente é sabido que os corpos temporários de água, resultados da precipitação intensa que caracteriza a estação chuvosa, têm sido adequados para a criação de mosquitos na maioria das "áreas de malária" e, em alguns casos, aumentam o risco da doença. Por isso, os valores da correlação apresentados na Tabela 5 demostram que os níveis de precipitação são importantes apenas se a água formar poças que persistam por tempo suficiente para o desenvolvimento do mosquito. Apesar da escala de dados não pemitir resultados que demostrem correlação forte, os mesmos demostram que existe tendência estatisticamente significativa da correlação entre a precipitação e a malária tanto com a variação mensal da precipitação como da variação sazonal. 
Por outro lado, a evolução da incidência da malária na cidade de Quelimane durante os 17 anos analisados demonstrou que a doença apresentou variação considerável, ou seja, apontou para instabilidade entre os 10\% (valor registrado em 2000) e 29\% (registrado em 2017) no que se refere à taxa de incidência. Apesar da tamanha variabilidade inter-anual, nota-se uma tendência de aumento dos casos de malária, o que, até certo ponto, indica maior dificuldade no controle da doença conforme os dados indicados na Figura 4.

Figura 4 - Gráfico da evolução do crescimento da população e casos de malária em Quelimane - 1980 á 2017

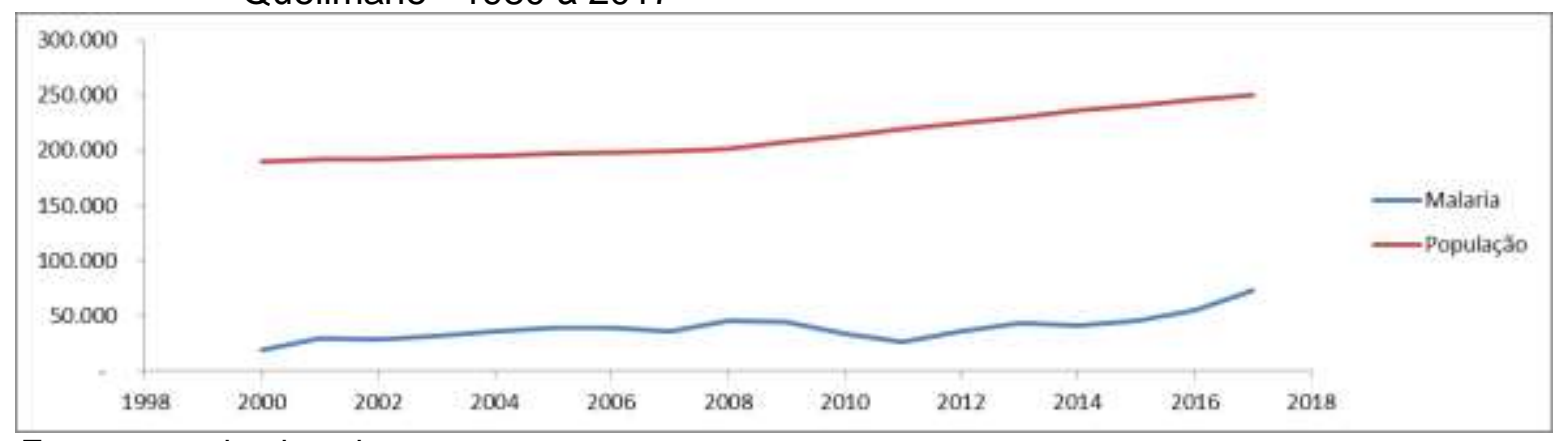

Fonte: organizado pelo autor.

De maneira geral, os anos de 2008, 2016 e 2017 apresentaram aumento, sobretudo no que se refere à maior percentagem da taxa de incidência durante a série em estudo, e os anos de 2000, 2002 e 2011 sinalizaram os menores valores percentuais.

Ainda no contexto da evolução da doença, observando a tendência do crescimento da população de 2000 a 2017, por meio dos dados projetados pelo (INE) Instituto Nacional de Estatísticas (1997, 2007 e 2013), e o aumento dos casos registrados da malária, a Figura 4 demonstra que, apesar da grande variabilidade que se nota anualmente, ou seja, a variabilidade inter-anual nos aspectos referentes à incidência da doença, a malária tende a aumentar com o crescimento da população na cidade de Quelimane.

Em suma, por meio das análises dos resultados apresentados, torna-se importante mencionar que o estudo da variabilidade da temperatura e da precipitação pluviométrica de um determinado espaço geográfico e a análise das suas séries históricas têm sido utilizados em várias pesquisas como um instrumento para avaliar as dinâmicas do comportamento climático e as consequências decorrentes deste fenômeno em diversos países, como exemplo, Brasil, no Oeste de África e em alguns países da Ásia e na África Subsaariana.

Os resultados sobre a tendência de variação da temperatura e precipitação apresentados neste artigo são comprovados com estudos realizados por Queface; Tadross (2009) e (INGC) Instituto Nacional de Gestão de Calamidades (2009) a respeito das mudanças climáticas em Moçambique, quando sustentam que, na região centro do país, onde Quelimane se localiza, registraram-se temperaturas máximas médias de $30^{\circ} \mathrm{C}$ antes de 
1990, e aumentos significativos subsequentemente durante o período de 45 anos, ou seja, de 1960 a 2005.

Sobre a precipitação, Queface e Tadross (2009) referem que pela lacuna e credibilidade dos dados existentes em Moçambique não se registraram tendências significativas na alteração da pluviosidade no período de 1960 a 2005, o que por um lado indica a aparente homogeneidade na variação da precipitação na cidade de Quelimane. Apesar disso, este autor chama a atenção para indícios da diminuição da precipitação anual durante os 45 anos que utilizou para sua análise, e, este aspecto foi comprovado com a tendência da variabilidade inter-anual da precipitação apresentada na Tabela 2.

Por meio de métodos estatísticos utilizados por Pereira e Rebelo (2000) no município de Maranhão, análises feitas por Coutinho (2015) em Manaus, e resultados da pesquisa desenvolvida por Sitoe (2017), as correlações obtidas neste artigo foram positivas tanto na variação mensal como na variação sazonal da temperatura com a malária, bem como na variação da precipitação com a malária, o que indica influências da variabilidade climática na ocorrência da doença.

\section{CONSIDERAÇÕES FINAIS}

A ocorrência da malária na cidade de Quelimane está fortemente associada a fatores de ordem natural, sobretudo a variabilidade da temperatura e precipitação pluviométrica porque a sua variação se correlaciona positivamente com os eventos da doença.

A incidência de malária durante os 17 anos mostrou uma associação positiva com a temperatura e com a precipitação, sendo que a temperatura é favorável em quase todo o ano e a precipitação exerce influência imediata, contribuindo de forma significativa na escala sazonal e anual.

As correlações encontradas neste artigo não foram fortes pela escala de dados disponível, uma vez que, para se estabelecer correlações fortes entre a variabilidade da temperatura e precipitação na transmissão da malária, deve ser levado em consideração não apenas as variações anuais das médias de temperatura, mas também, mais importante, a amplitude da temperatura, a variabilidade mensal e interanual das chuvas e os dados de registros da malária na escala diária ou semanal.

Finalmente torna-se fundamental sustentar que a relação entre os fatores climáticos com a malária não é estabelecida de "forma direta", e existem outros fatores que devem ser levados em consideração, o que sugere futuros estudos voltados para a influência dos fatores socioeconômicos e da política pública.

As fraquezas deste estudo estão relacionadas com a qualidade dos dados, tanto os dados de saúde como os dados climáticos. Por isso, recomenda-se que as autoridades 
públicas em Moçambique melhorem a forma de recolha e organização da informação respeitando a escala diária em seus bancos de dados, pois estudos aprofundados nesta temática são necessários para o estabelecimento de políticas públicas eficientes.

\section{REFERÊNCIAS}

ADU-PRAH, S.; KOFFI, T. E. Spatiotemporal analysis of climate variability impacts on malária prevalence in Ghana. Applied Geography, Oxford, GB, v. 60, p. 266-273, Jun. 2015. Disponível em: https://www.sciencedirect.com/science/article/abs/pii/S0143622814002471. Acesso em: 8 set. 2018.

BAUTISTA, C. T.; CHAN, A. S.; RYAN, J. R.; CALAMPA, C.; ROPER, M. H.; HIGHTOWER, A. W.; MAGILL, A. J. Epidemiology and spatial analysis of malaria in the Northern Peruvian Amazon. American Journal of Tropical Medicine and Hygiene, Mclean, VA, v. 75, n. 6. p. 1216-1222, 2006. Disponível em: https://www.ncbi.nlm.nih.gov/pubmed/17172396. Acesso em: 4 maio 2019.

BHATTACHARYA, S.; SHARMA, C.; DHIMAN, R. C.; MITRA, A. P. Climate change and malaria in India. Current Science, Bangalore, IN, v. 90, n. 3, p. 369-374, 2006.

BRAGANZA, K.; KAROLY, D.; HIRST, A.; MANN, M.; STOTT, P.; STOUFFER, R.; TETT, S. Simple indices of global climate variability and change: part I-variability and correlation structure. Climate Dynamics, Berlin, DE, v. 20, n. 5, p. 491-502, 2003. Disponível em: https://link.springer.com/article/10.1007/s00382-002-0286-0. Acesso em: 6 de ago. 2019.

BRIËT, O. J.; VOUNATSOU, P.; GUNAWARDENA, D. M.; GALAPPATHTHY, G. N.; AMERASINGHE, P. H. Temporal correlation between malaria and rainfall in Sri Lanka. Malaria Journal, Liverpool, UK, v. 7, n. 1, p. 77, 2008.

CAPINHA, C. D. S. O mosquito vetor da Malaria Anopheles Atroparvus, Van Thiel. 1927: adequabilidade de habitat em Portugal continental e potenciais alterações futuras do seu espaço climático. 2009. Dissertação de (Mestrado em Sistemas de Informação Geográfica e Modelação Territorial Aplicados ao Ordenamento) - Universidade de Lisboa, PT, Lisboa, 2009.

CHRISTOFOLETTI, A. Procedimentos de análise utilizados no estudo da precipitação. Geociências, São Paulo, v. 11, n. 6, p. 75-98, 1992.

CONFALONIERI, U. Variedade climática, vulnerabilidade social e saúde no Brasil. Terra Livre, São Paulo, ano 19, v. 1, n. 20, p. 193-204, 2003.

CORDEIRO, C. E. S.; FILOMENO, C. R. M.; COSTA, C. M. A. D.; COUTO, Á. A. R. D. Perfil epidemiológico da malária no Estado do Pará em 1999 com base numa série histórica de dez anos (1989-1999). Informe Epidemiológico do SUS, Brasília, DF, v. 11, n. 2, p. 69-77, 2002.

COSTA, M. da C.; TEIXEIRA, M. G. A concepção de "espaço" na investigação epidemiológica. Cadernos de Saúde Pública, Rio de Janeiro, RJ, v. 15, p. 271-279. 1999.

COUTINHO, P. E. G. Análise do padrão de incidência da malaria e sua relação com fatores climáticos e hidrológicos em escala sub-regional e local na Bacia do Rio Negro. 2015. Tese (Doutorado em Clima e Ambiente) - Instituto Nacional de Pesquisas da Amazônia; Universidade Estadual do Amazonas, Manaus, AM, 2015.

EPSTEIN, P. Study on the impact of climate change on disaster risk in Mozambique. In: NUSSBAUMER, P.; PATT, A. (ed.). INGC synthesis report on climate change: first draft. Maputo, MZ: National Institute for Disaster Management, 2009. p. 53-56. 
ERNST, K. C.; ADOKA, S. O.; KOWUOR, D. O.; WILSON, M. L.; JOHN, C. C. Malaria hotspot areas in a highland Kenya site are consistent in epidemic and non-epidemic years and are associated with ecological factors. Malaria Journal, Liverpool, UK, v. 5, n. 1, p. 7888, 2006.

FIGUEIREDO FILHO, D. B.; SILVA JÚNIOR, J. A. D. Desvendando os mistérios do coeficiente de correlação de Pearson (r). Política Hoje, Recife, PE, v. 18, n. 1, p. 115-146, 2009.

FRANÇA, T.; SANTOS, M.; FIGUEROA-VILLAR, J. Malária: Aspectos Históricos e Quimioterapia. Química Nova, São Paulo, SP, v. 31, n. 5, p. 1271-1278, 2008.

GEMPERLI, A.; VOUNATSOU, P.; SOGOBA, N.; SMITH, T. Malaria mapping using transmission models: application to survey data from Mali. American Journal of Epidemiology, Baltimore, MD, v. 163, n. 3, p. 289-297, 2005.

HASHIZUME, M.; TERAO, T.; MINAKAWA, N. The Indian Ocean Dipole and malaria risk in the highlands of western Kenya. Proceedings of the National Academy of Sciences, Allahabad, IN, v. 106, n. 6, p. 1857-1862, 2009.

IMPOINVIL, D. E.; CARDENAS, G. A.; GIHTURE, J. I.; MBOGO, C. M.; BEIER, J. C. Constant temperature and time period effects on Anopheles gambiae egg hatching. Journal of the American Mosquito Control Association, Fresno, CA, v. 23, n. 2, p. 124, 2007.

INSTITUTO NACIONAL DE ESTATÍSTICA. Censo 1997: IV recenseamento geral da população e habitação. resultados definitivos: Maputo: INE, 1997.

INSTITUTO NACIONAL DE ESTATÍSTICA. Censo 2007: IV recenseamento geral da população e habitação: resultados definitivos. Maputo: INE, 2007.

INSTITUTO NACIONAL DE ESTATÍSTICA. Estatística do Distrito de Quelimane. Maputo: INE, 2013.

INSTITUTO NACIONAL DE GESTÃO DE CALAMIDADES. Estudo sobre o impacto das alterações climáticas no risco de calamidades em Moçambique. Maputo, MOZ: INGC, 2009.

INSTITUTO NACIONAL DE METEOROLOGIA DE MOÇAMBIQUE. Compilação dos dados climáticos da Província da Zambézia. Quelimane: INAM, 2018.

LIMA NETO, J. E. Geografia e saúde. 2000. Dissertação (Mestrado em Geografia) Universidade de São Paulo, SP, São Paulo, 2000.

MABASO, M. L.; VOUNATSOU, P.; MIDZI, S.; SILVA, J.; SMITH, T. Spatio-temporal analysis of the role of climate in inter-annual variation of malaria incidence in Zimbabwe. International Journal of Health Geographics, New York, NY, v. 5, n. 1, 20, p. 1-9, 2006.

MABUNDA, S. J. The epidemiology and the burden of malaria in Mozambique. 2006. Thesis (PhD in Medicine and Surgery) - University of Barcelona, Barcelona, ES, 2006.

MENENDEZ, C. Malaria during pregnancy: a priority area of malaria research and control. Parasitology Today, Amsterdam, NL, v. 11, n. 5, p. 178-183, 1995.

MOÇAMBIQUE. Ministério da Saúde. Documento estratégico para o controlo da malária em Moçambique, julho 2006 - 2009. Maputo: MISAU, 2006.

MOÇAMBIQUE. Ministério da Saúde. Programa Nacional de Controlo da Malária. Relatório da Província da Zambézia 2017. Quelimane: MISAU, 2018.

MOÇAMBIQUE. Ministério para Coordenação da Ação Ambiental. Relatório de avaliação participativa da vulnerabilidade a mudanças climáticas. Maputo: MICOA, 2004.

NUNES, L. H.; LOMBARDO, M. A. A questão da variabilidade climática: uma reflexão crítica. Revista do Instituto Geológico, São Paulo, v. 16, n. 1/2, p. 21-31, 1995. 
OESTERHOLT, M. J. A. M.; BOUSEMA, J. T.; MWERINDE, O. K.; HARRIS, C.; LUSHINO, P.; MASOKOTO, A.; DRAKELEY, C. J. Spatial and temporal variation in malaria transmission in a low endemicity area in northern Tanzania. Malaria Journal, Liverpool, UK, v. 5 , n. 1, p. 1-5, 2006.

PEITER, P. C. A geografia da saúde na faixa de fronteira continental do Brasil na passagem do milênio. 2007. Tese (Doutorado em Geografia) - Universidade Federal do Rio de Janeiro, RJ, 2007.

PEREIRA, Y., REBELO, J. M. Espécies de Anopheles no município de Pinheiro (Maranhão), área endêmica de malária. Revista da Sociedade Brasileira de Medicina Tropical, Uberaba, MG, v. 33, n. 5, p. 443-450, 2000.

PETERNELLI, L. Regressão linear e correlação. 2015. Disponível em: http://www.dpi.ufv.br/ peternelli/inf162.www.16032004/materiais/CAPITULO9.pdf. Acesso em: 3 maio 2019.

QUEFACE, A.; TADROSS, M. Study on the impact of climate change on disaster risk in Mozambique. In: ASANTE, K.; BRITO, R., BRUNDRIT, G.; EPSTEIN, P.; FERNANDES, A.; MARQUES, M. R.; MAVUME, A.; METZGER, M.; PATT, A.; QUEFACE, A.; TADROSS, M.; BRITO, R. (ed.). Main report: INGC climate change report: study on the impact of climate change on disaster risk in Mozambique Maputo, MZ: Ministry of State Administration, 2009. p. 1-44.

QUEIROZ, A. T.; COSTA, R. A. Caracterização e variabilidade climática em series de temperatura, umidade relativa do ar e precipitação em Ituiutaba - MG. Caminhos de Geografia, Uberlândia, MG, v. 13, n. 43, p. 346-357, 2012.

SAÚTE, F.; APONTE, J.; AHMEDA, J.; ASCASO, C.; VAZ, N.; DGEDGE, M.; ALONSO, P. Malaria in southern Mozambique: incidence of clinical malaria in children living in a rural community in Manhica district. Transactions of the Royal Society of Tropical Medicine and Hygiene, London, GB, v. 97, n. 6, p. 655-660, 2003.

SILVESTRE, M.; LIMA NETO, J.; FLORES, E. Critérios estatísticos para definir anospadrão: uma contribuição à climatologia geográfica. Revista Formação, Presidente Prudente, SP, v. 2, n. 20, p. 23-53, 2013.

SITOE, G. A. Risco e vulnerabilidades socioambientais à Malária em Quelimane Zambézia, Moçambique. 2017. Tese (Doutorado em Geografia) - Universidade Federal do Paraná, Curitiba, 2017.

SNOW, R.; GILLES, H. The epidemiology of malaria. In: WARRELL, D. A.; GILLES, H. M. (ed.). Essential malariology. New York: Oxford University Press, 2002. p. 85-106.

SUTHERST, R. W. Global change and human vulnerability to vector-borne diseases. Clinical Microbiology Reviews, Washington, US, v. 17, n. 1, p. 136-173, 2004.

THOMSON, M. C.; MASON, S. J.; PHINDELA, T.; CONNOR, S. J. Use of rainfall and sea surface temperature monitoring for malaria early warning in Botswana. American Journal of Tropical Medicine and Hygiene, Mclean, VA, v. 73, n. 1, p. $214-221,2005$.

UELE, D. Impactos dos modos de variabilidade climática no cultivo do milho em regiões de sequeiro: uma abordagem aplicada a sustentabilidade agrícola na região sul de Moçambique. 2013. Dissertação (Mestrado em Práticas de Desenvolvimento Sustentável) Universidade Federal Rural do Rio de Janeiro, Seropédica, RJ, 2013.

VILELA JUNIOR, G. B. Estatística: teste t student. 2019. Disponível em: http://www.cpaqv.org/estatistica/teste_t.pdf. Acesso em: 4 abr. 2019.

WEBBER, R. Communicable disease epidemiology and control: a global perspective. Cambridge, MA: CABI Publishing, 2004. 
WORLD HEALTH ORGANIZATION. From malaria control to malaria elimination: a manual for elimination scenario planning. Geneva, SW: WHO, 2014.

WORLD HEALTH ORGANIZATION. World malaria report. Geneva, SW: WHO, 2013.

XAVIER, T. M. B. S.; SILVA, J. F. da; REBELLO, E. R. G. A técnica dos quantis e suas aplicações em meteorologia, climatologia e hidrologia, com ênfase para as regiões brasileiras. Brasília: Thesaurus, 2002.

XAVIER, T. M. B. S.; XAVIER, A. S. F. Caracterização de períodos secos ou excessivamente chuvosos no Estado do Ceará através da técnica dos quantis: 1964-1998. Revista Brasileira de Meteorologia, São José dos Campos, SP, v. 14, n. 2, p. 63-78. 1999.

Recebido: agosto de 2019. Aceito: dezembro de 2019. 